\title{
Reitunfälle - Fakten und Abläufe bei der Rettung
}

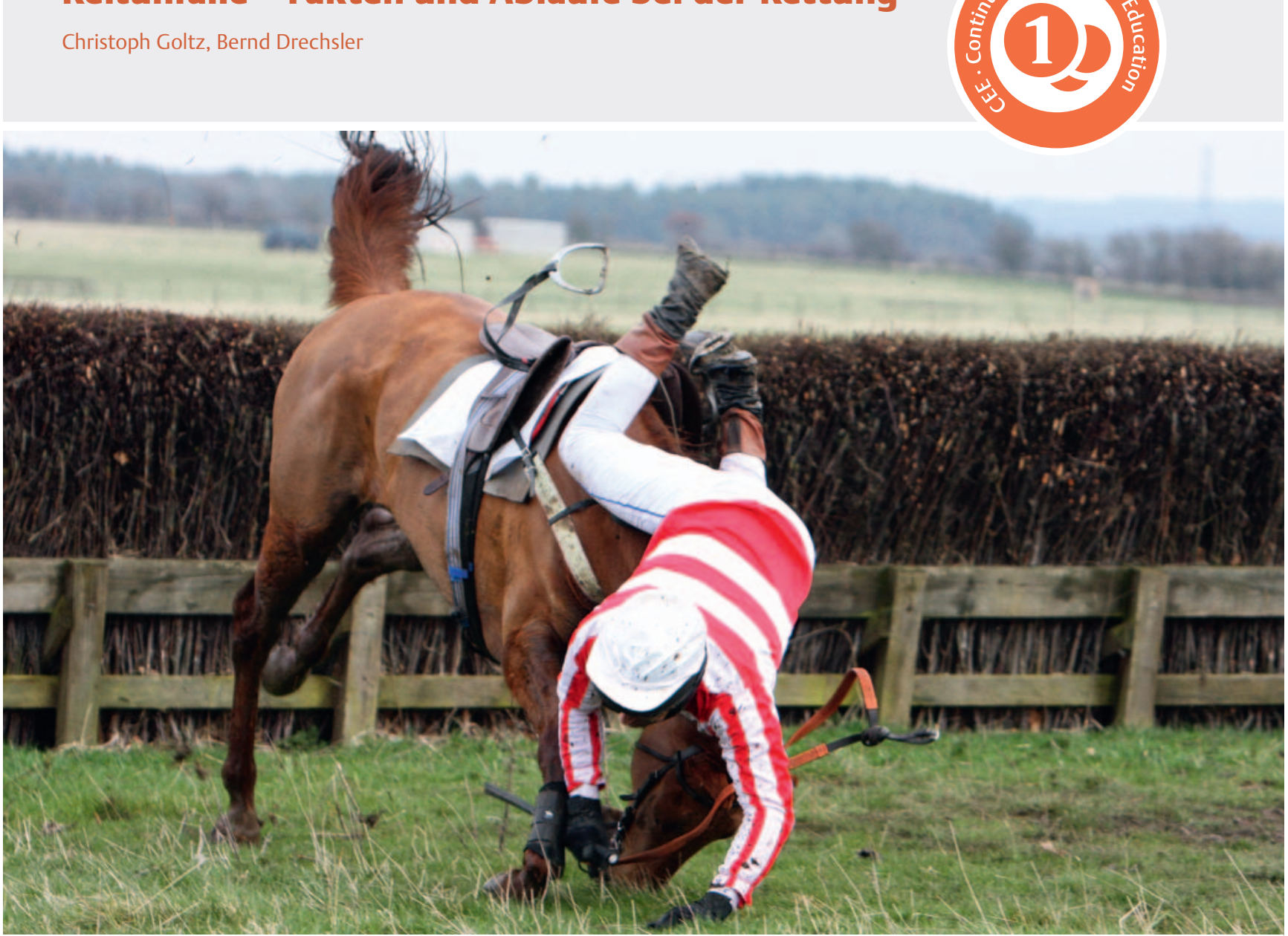

Quelle: Verity Johnson/AdobeStock.com

Alles Glück dieser Erde liegt auf dem Rücken der Pferde - so der Volksmund. Um auch im Unglücksfall im Bereich des Pferdesports gewappnet zu sein, haben wir in diesem Artikel einige Besonderheiten und Fakten im Umgang mit Reitunfällen zusammengetragen.

\section{Statistik}

Der Reitsport erfreut sich in Deutschland größter Beliebtheit ( $\triangleright$ Abb. 1). Die Mitgliederzahl der Deutschen Reiterlichen Vereinigung lag 2017 bei 687036 Personen - ca. $80 \%$ davon Frauen und Mädchen. Nach einer Studie der Allensbacher Markt- und Werbeträgeranalyse (AWA) aus 2016 waren 3,89 Mio. Menschen in Deutschland Reiter, worunter 1,25 Mio. die Sportart intensiv betrieben. Auch dabei lag der Anteil des weiblichen Geschlechts bei $78 \%$ [1]. Einer von 10000 Reitern erleidet jährlich einen tödlichen Reitunfall [2] - jeder Fünfte verletzt sich statistisch gesehen im Lauf seiner Reitkarriere ernsthaft [3]. Insbesondere die Altersgruppe der 8- bis 18-Jährigen erleidet
Reitunfälle, wobei die schwer bis schwerstverletzten Patienten meist älter sind. Gemäß der weitaus höheren Zahl von Mädchen und Frauen im Reitsport ist die überwiegende Anzahl der Verletzten weiblich [4, 5]. Im Vergleich zu Alpinsportarten, American Football oder Motorsport ist das Risiko schwerer Verletzungen beim Reitsport höher [5, 6].

\section{Unfallmechanismen}

Ein sicherlich wesentlicher Aspekt der Unfallhäufigkeit im Reitsport: Pferde sind Lebewesen und kein Sportgerät. 


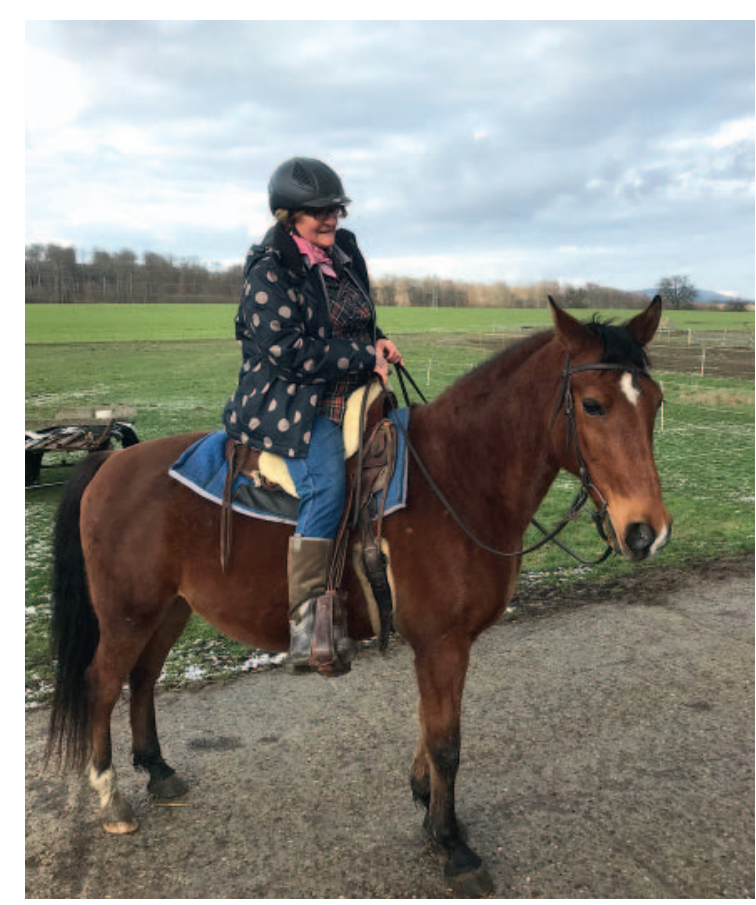

- Abb. 1 Freizeitreiterin auf ihrem Pferd.

\section{Merke}

Pferde sind selbstständig denkende Fluchttiere, wiegen im Schnitt 500 kg, können mit einer Kraft von bis zu einer Tonne treten und eine Geschwindigkeit von $65 \mathrm{~km} / \mathrm{h}$ erreichen.

Der Kopf des Reiters befindet sich je nach Pferd in ca. 2,7 m Höhe [5, 6]. Diese Fakten und die daraus resultierende kinetische Energie bergen bei Unfällen ein nicht unerhebliches Risiko. Unfälle geschehen auch im Umgang mit den Tieren: bei der Pflege, beim Füttern oder bei Transporten. Der häufigste Unfallmechanismus mit ca. 80 \% ist der Sturz vom Pferd, gefolgt von:

- Pferdetritt

- Bissen

- Verletzungen durch das Zaumzeug

- Sturz des Pferds auf den Reiter

- Anprall von Pferd und Reiter gegen ein Hindernis

- Quetschungen/Stößen

- Pferd überrennt Reiter [4-6]

\section{Verletzungsmuster}

Verletzungen beim Reitsport sind aufgrund der Vielzahl möglicher Unfallmechanismen extrem heterogen. In über 80 \% der Unfälle kommt es zu Einzelverletzungen [5] - häufig betroffen ist die Hand (z. B. durch das Zaumzeug) oder der menschliche Fuß (Tritt des Pferdes). Pferdetritte, v. a. gegen den Kopf, können zu schwersten Verletzungen - wie Impressionsfrakturen des Schädels führen. Gleiches gilt für Pferdetritte gegen jedwede Re- gion des menschlichen Körpers [5, 7]. Verletzungen beim Sturz vom Pferd betreffen meist folgende Bereiche:

- oberen Extremitäten

- Kopf

- Wirbelsäule

- Körperstamm

- Becken $[5,6]$

Je nach Unfallkinetik resultieren Mehrfachverletzungen, Polytrauma und mögliche Todesfolge. Stürze bei geringem Tempo auf weichen Untergrund führen i. d. R. zu leichteren Verletzungen als Stürze bei hohem Tempo auf harten Grund oder auf Gegenstände. Zu besonders schweren Traumata kommt es, wenn Reiter und Pferd gemeinsam stürzen und der Mensch unter dem Tier begraben oder von dessen Hufen getroffen wird [5, 8].

\section{Besonderheiten bei der Rettung}

Speziell auf den Reitunfall ausgelegte Leitlinien existieren unserer Recherche nach nicht - die Heterogenität der möglichen Szenarien macht ein standardisiertes Vorgehen wohl nicht möglich. Gängige Kurskonzepte wie das prioritätenorientierte Vorgehen nach $\mathrm{PHTLS}^{\circledR}$ bieten sich als Grundvorbereitung an - Notärzte und Rettungspersonal finden Fortbildungskurse u. a. bei der Hamburger AG Reitsicherheit. Wie so oft in der präklinischen Notfallmedizin sind ein gesundes Maß an Menschenverstand, das MacGyver-Gen, die vorhandene Ausrüstung und die gängigen, allgemeingültigen Leitlinien hilfreich, Reitunfälle abzuarbeiten. Dennoch gibt es einige Gedankengänge bzw. Probleme, die wir im Folgenden darstellen.

\section{Problem Unfallort}

Unfälle im Reitsport ereignen sich zum einen an gut zugänglichen Orten wie Reithallen, Reitplätzen oder Ställen, zum anderen an schwer erreichbaren Plätzen im freien Gelände - die Rettung wird dann zu einer enormen Herausforderung. Handyortungen können dem Leitstellendisponenten bei langen Suchaktionen helfen, den Einsatzort zu definieren. Auch der Abtransport des Patienten kann in diesen Fällen schwierig und zeitraubend sein. Wenn es der Zustand des Patienten erfordert und ein geeigneter Landeplatz vorhanden ist, sollte frühzeitig ein Rettungshubschrauber eingesetzt und vor dessen Landung sollten die Pferde gesichert werden.

\section{Problem Eigensicherung}

Pferde sind zwar scheue Fluchttiere, können aber nach einem Reitunfall den Patienten und die Retter in Gefahr bringen, z. B. bei einem Unfall in einer geschlossenen Pferdebox oder wenn weitere Pferde im näheren Umfeld sind. Um die Tiere nicht zu erschrecken, empfiehlt es sich bei Annäherung zur Einsatzstelle die Sondersignalanlage bzw. das Martinshorn abzuschalten und die letzten Meter 
langsam zurückzulegen. Das Wichtigste ist zunächst die Eigensicherung, dann folgt der Überblick über den Einsatzort:

- Lage des/der Verletzten

- Sicherung des Pferds/der Pferde

- sonstige Gefahrenquellen

Cave

Die Annäherung an ein Pferd sollte langsam von vorne oder von der Seite erfolgen - niemals von hinten.

Idealerweise sichert eine pferdekundige Person die Tiere und verständigt bei Bedarf einen Tierarzt.

\section{Problem Anamnese und Unfallhergang}

Ein Reitunfall ist häufig schwer rekonstruierbar. Bei bewusstlosem Reiter auf dem Boden gibt es zunächst wenig Anhaltspunkte über den Unfallhergang ( $\triangleright$ Abb. 2). Ist der Reiter vom Pferd gestürzt? Wie hoch war das Tempo? Ist der Reiter gegen ein Hindernis geprallt? Ist das Pferd auf den Reiter gestürzt? Wurde der Reiter im Steigbügel festhängend mitgeschleift und/oder sekundär von den Hufen getroffen? Selbst bei Bewusstsein des Reiters kann dieser oft wenig Auskünfte geben. Eine kurze Befragung möglicher Unfallzeugen und eine genaue Betrachtung des Unfallorts können Aufschlüsse über Unfallhergang und mögliche Verletzungsmuster liefern.

\section{Schockraumalarmierung: ja oder nein?}

In der Infobox sind die Kriterien für eine Schockraumalarmierung nach der S3-Leitlinie Polytrauma/Schwerverletzten-Behandlung der Deutschen Gesellschaft für Unfallchirurgie aufgeführt.

\section{INFO}

Bei folgenden Verletzungen soll das Trauma-/Schockraumteam aktiviert werden:

- systolischer Blutdruck unter $90 \mathrm{mmHg}$ (altersadaptiert bei Kindern) nach Trauma

- Vorliegen von penetrierenden Verletzungen der Rumpf-Hals-Region

- Vorliegen von Schussverletzungen der Rumpf-Hals-Region

- GCS unter 9 nach Trauma

- Atemstörungen/Intubationspflicht nach Trauma

- Frakturen von mehr als 2 proximalen Knochen

- instabiler Thorax

- Beckenfrakturen

- Amputationsverletzung proximal der Hände/Füße

- Querschnittsverletzung

- offene Schädelverletzungen

- Verbrennungen $>20 \%$ und Grad $\geq 2 b$

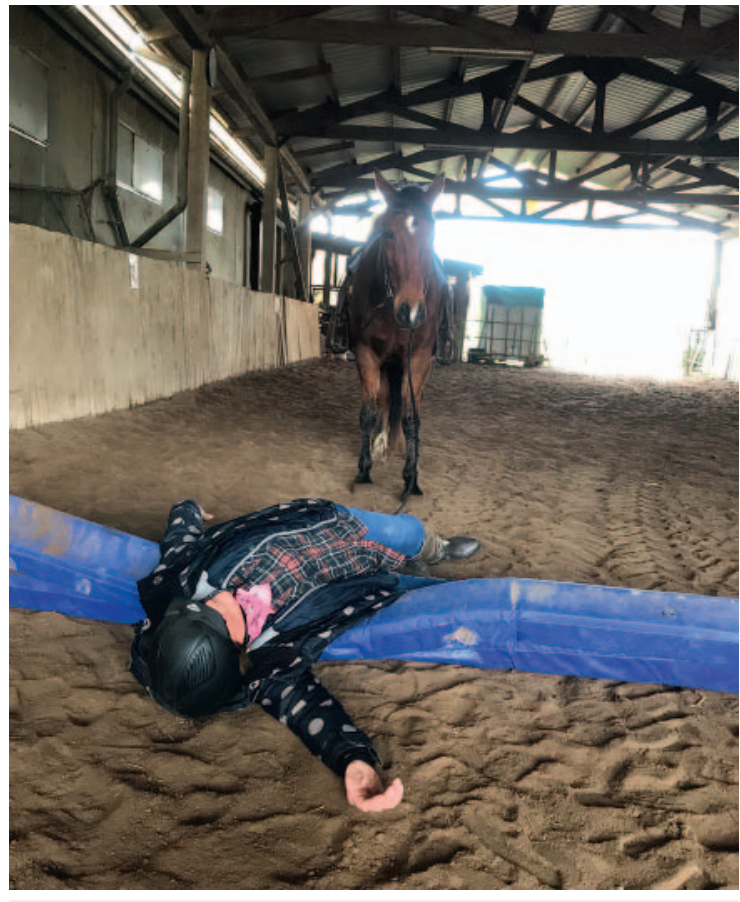

- Abb. 2 Reiterin stürzt von einem Pferd auf ein Hindernis (nachgestellte Situation).

Zusätzliche Kriterien zur Alarmierung:

- Sturz aus über 3 Metern Höhe

- Verkehrsunfall (VU) mit

- Frontalaufprall mit Intrusion von mehr als $50-75 \mathrm{~cm}$

- einer Geschwindigkeitsänderung von $\Delta>30 \mathrm{~km} / \mathrm{h}$

- Fußgänger-/Zweiradkollision

- Tod eines Insassen

- Ejektion eines Insassen

Die Indikation zur Schockraumaktivierung anhand bestehender Verletzungen ist auch bei einem Reitunfall relativ leicht zu stellen - sei es ein Sturz, ein Tritt oder ein anderer Unfallmechanismus. Schwieriger wird es ohne „harte“ Verletzungskriterien und bei schwer einschätzbarer Unfallkinetik. Da ein Pferd bereits im Trab 10-20 km/h und im Galopp bis zu 65 km/h erreicht, ist das Kriterium der Geschwindigkeitsänderung relativ schnell erreicht. Doch woher wissen wir, wie hoch das Tempo war? Fällt der Reiter vom Rücken des Pferds eine Böschung hinab, können 3 Meter schnell erreicht werden.

\section{Merke}

Unserer Meinung nach ist in diesen Fällen die Indikation zur Schockraumalarmierung großzügig, aber auch mit gesundem Menschenverstand zu stellen. 


\section{Vorgehen bei Reitunfällen}

\section{Allgemeines Vorgehen}

- Umsichtige Anfahrt

- Auf Eigensicherung achten

- Vorgehen nach prioritätenorientiertem ABCDE-Schema

- Monitoring mit Pulsoxymetrie, RR-Messung, EKG-Ableitung

- Befragen des Patienten und der Unfallzeugen nach dem Unfallhergang, Symptome/Schmerzen, Allergien, Eigenmedikation, Vorerkrankungen, mögliche Schwangerschaft, letzte Mahlzeit, sonstige Besonderheiten

\section{Vorgehen bei Reitunfällen mit Sturz vom Pferd}

Bei diesem dynamischen Unfallmechanismus muss der Unfallhergang möglichst genau eruiert werden. Bei polytraumatisierten Patienten empfiehlt sich das Vorgehen nach der S3-Leitlinie Polytrauma/Schwerverletzten-Behandlung der Deutschen Gesellschaft für Unfallchirurgie. Zunächst erfolgt nach bzw. während des genannten allgemeinen Vorgehens die Immobilisation des Patienten. Bei vitaler Bedrohung muss man das Vorgehen zur Lösung des bestehenden Problems prioritätenorientiert abändern. Mittels der Zweihelfermethode wird unter Stabilisierung der Halswirbelsäule der Reiterhelm abgenommen und ein Stifneck angelegt. Falls nötig: Sauerstoffgabe, Notfallnarkose und Atemwegssicherung, Entlastung eines (Spannungs-)Pneumothorax und Blutungskontrolle bei bestehender Indikation. Der Patient wird dann, z. B. mittels Vakuummatratze, weiter immobilisiert. Anlegen einer Beckenschlinge bei Verdacht auf eine Beckenverletzung. Man untersucht den Rücken des Patienten beim Drehen („Log-roll“-Manöver) zur Umlagerung. Wenn möglich erfolgt im vorgeheizten RTW die komplette Entkleidung und körperliche Untersuchung. Regelmäßige Wiederholung des ABCDE-Schemas. Das Verletzungsmuster bestimmt das weitere Vorgehen. Sinnvoll ist, in einem kurzen „Team Time-out“ die relevanten Fakten und Befunde zusammenzutragen, Probleme zu definieren und das weitere Vorgehen festzulegen (10-für10-Prinzip). Je nach Befund folgt die Anlage mind. eines periphervenösen Zugangs, Flüssigkeits- und Medikamentengabe. Schienung und gegebenenfalls Reposition vorhandener Extremitätenverletzungen unter adäquater Analgosedierung. Die präklinische Zeit muss v. a. beim polytraumatisierten Patienten kurz sein (golden hour of shock). Je nach lokalen Gegebenheiten, Verletzungsmuster und Zustand des Patienten informiert man die nächstgelegene, geeignete Zielklinik. Ist ein Zeitgewinn zu erwarten und der Patient ausreichend stabil, kann der Transport mittels RTH erfolgen. Vor Transportbeginn hilft ein erneutes Team Time-out, sich einen gemeinsamen Überblick über den Zustand des Patienten und die Vollständigkeit der getroffenen Maßnahmen zu verschaffen sowie Ablauf oder Besonderheiten für den Transport zu kommunizieren.

\section{FALLBEISPIEL 1}

Sturz vom Pferd

Alarmierung NEF und RTW: weiblich, 30 Jahre, bewusstlos. Die Einsatzkräfte erreichen einen Reitsportplatz und schalten die Sondersignalanlage ab. Die Patientin liegt in stabiler Seitenlage. Das Pferd steht abseits - angeleint und betreut. Ein Augenzeuge berichtet: Sturz bei hohem Tempo und Aufprall mit dem Kopf (ohne Helm) auf die Stange der Bande. Der Erstbefund zeigt eine tief bewusstlose Frau mit einer GCS von 3, seitlich am Kopf mit Kopfplatzwunde. Atmung tief und gleichmäßig, seitengleich vesikuläres Atemgeräusch. Pulse sind peripher gut tastbar. Eine Pupillendifferenz deutet auf ein schweres Schädel-Hirn-Trauma hin. Keine weiteren Verletzungen. Der Notarzt stabilisiert die Halswirbelsäule manuell in Neutralposition des Kopfes. Der Rettungsassistent des NEF bereitet die Intubation und die notwenigen Medikamente vor. Es erfolgt die Anlage eines Stifneck - der Kopf wird dennoch weiterhin in Neutralposition gehalten und zeitgleich Sauerstoff hochdosiert über eine Maske verabreicht. Im Bereich der Wirbelsäule findet sich keine Stufenbildung. Die Patientin wird an den Monitor angeschlossen $\left(\mathrm{SO}_{2}\right.$, RR-Messung, EKG) und in Rückenlage gebracht. Es folgen: Legen eines i.v. Zugangs, Intubationsnarkose, Ergänzung des Monitorings um die Kapnografie und Umlagerung der Patientin auf eine Vakuummatratze in den vorgeheizten RTW. Abstimmung des weiteren Vorgehens im kurzen Team Time-out. Wiederholung des ABCDE-Schemas, komplette Entkleidung der Patientin und vollständiger Bodycheck - ein zweiter i. v. Zugang wird gelegt und das Schockraumteam der nahegelegenen Uniklinik alarmiert. Während des Anmodellierens und Absaugens der Vakuummatratze werden im Team Time-out alle Befunde, Probleme und Maßnahmen aufgezählt und auf Vollständigkeit geprüft. Es folgt der Transport in die Klinik.

\section{Vorgehen bei Trittverletzungen durch das Pferd}

Die Behandlung von Trittverletzungen ähnelt in den Grundzügen dem oben Beschriebenen. Die betroffene Körperregion ist meist leichter abzugrenzen - die Verletzungsschwere ist jedoch nicht zu unterschätzen.

\section{Merke}

Angesichts der Trittkraft des Pferdes auf eine relativ kleine Fläche ist bis auf Weiteres von der Verletzung tiefer liegender Strukturen auszugehen. 


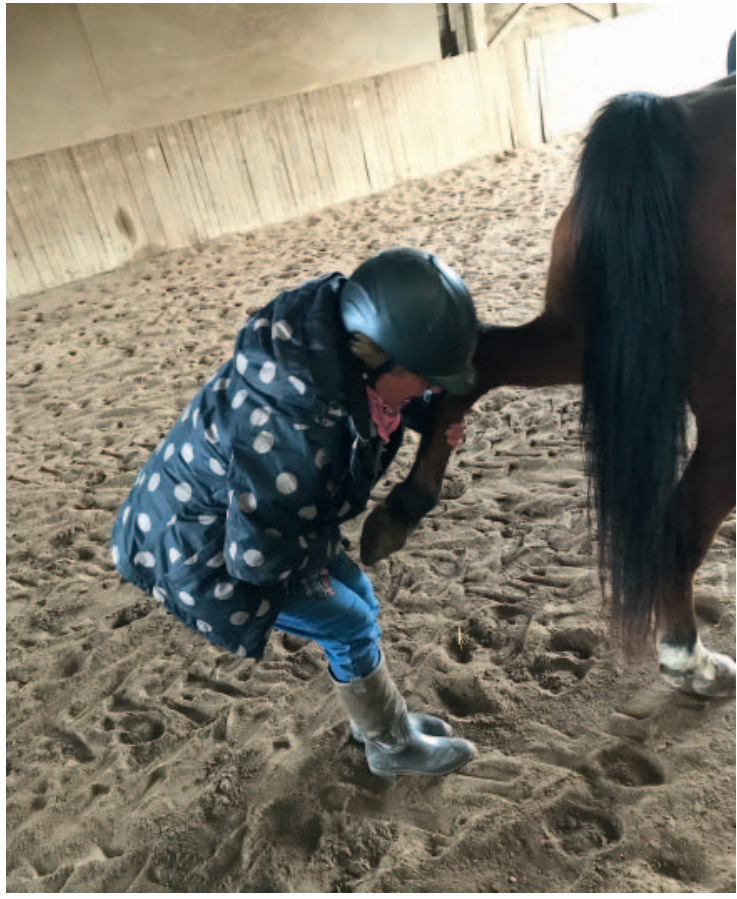

- Abb.3 Pferdetritt in den Bauch (nachgestellte Situation).

Selbst bei äußerlich dezenten Prellmarken kann es sich um eine lebensbedrohliche Verletzung handeln - Tritte gegen Thorax oder Abdomen führen nicht selten zu Verletzungen der inneren Organe ( $\triangleright$ Abb. 3). Um auch multiple Trittverletzungen zu erkennen, sollte die Untersuchung vollständig am komplett entkleideten Patienten erfolgen.

\section{FALLBEISPIEL 2}

\section{Pferdetritt Abdomen}

Alarmierung NEF und RTW: männlich, 54 Jahre. Bei einem Pferdehof findet der Rettungsdienst einen Mann vor, der bei der Hufpflege vom Pferd in den Bauch getreten wurde. Dieser hält das Hinzuziehen des Rettungsdienstes für übertrieben. Dem Notfallsanitäter fällt auf, dass der Mann sehr blass und zudem kaltschweißig ist. Nach geduldigem Zureden stimmt der Mann einer Untersuchung im RTW zu. Es findet sich eine deutliche Prellmarke im Bereich des rechten Oberbauchs. Der Bauch wirkt leicht gebläht, ist sehr drucksensibel - dem Patienten ist übel. Die Vitalparameter zeigen: systolischer Blutdruck 80 mmHG, Herzfrequenz 120/min bei Sauerstoffsättigung von $97 \%$. Im Team Time-out wird die Diagnose stumpfes Bauchtrauma mit hochgradigem Verdacht auf eine intraabdominelle Blutung gestellt. Der Patient stimmt aufgrund des Leidensdrucks einer Untersuchung im Krankenhaus zu. Wie im Time-out besprochen wird das Monitoring $\left(\mathrm{SO}_{2}\right.$, RR-Messung im 3-min-Intervall, EKG) vervollständigt. Das Team legt zwei großlumige Zugänge, substituiert Volumen und verabreicht Sauerstoff über eine Maske. Aufgrund zunehmend instabiler Kreislaufverhältnisse verabreicht der Notarzt $1 \mathrm{~g}$ Tranexamsäure. Nach erneutem kurzem Team Time-out wird der Patient, nach vorheriger Anmeldung, in den Schockraum der nahegelegenen Klinik der Schwerpunktversorgung transportiert.

\section{Fazit}

Reitunfälle sind vielschichtig und wegen des sehr dynamischen Unfallhergangs zunächst meist schwer einzuschätzen. Ein strukturiertes Vorgehen nach bewährten prioritätenorientierten Kurskonzepten (z. B. PHTLS) hilft, den Patienten optimal zu versorgen.

\section{KERNAUSSAGEN}

- Reitunfälle sind häufig - etwa jeder fünfte Reiter verletzt sich im Lauf seiner Reitkarriere ernsthaft.

- Pferde sind scheue Fluchttiere, wiegen im Schnitt $500 \mathrm{~kg}$, können eine Geschwindigkeit von $65 \mathrm{~km} / \mathrm{h}$ erreichen und mit einer Kraft von einer Tonne zutreten.

- Der häufigste Unfallmechanismus ist mit ca. $80 \%$ der Sturz vom Pferd, gefolgt von Pferdetritten.

- Bei einem Sturz vom Pferd kommt es am häufigsten zu Verletzungen der oberen Extremitäten und des Kopfs, der Wirbelsäule, des Körperstamms und des Beckens.

- Probleme bei Reitunfällen betreffen u. a. die unwegsamen Einsatzorte, die Eigensicherung und den schwer rekonstruierbaren Unfallhergang.

- Ein strukturiertes Vorgehen im Team, nach gängigen Kurskonzepten der Verletztenversorgung, hilft den Patienten optimal zu versorgen.

Interessenkonflikt

\section{Erklärung zu finanziellen Interessen}

Forschungsförderung erhalten: nein; Honorar/geldwerten Vorteil für Referententätigkeit erhalten: nein; Bezahlter Berater/interner Schulungsreferent/Gehaltsempfänger: nein; Patent/Geschäftsanteile/Aktien (Autor/Partner, Ehepartner, Kinder) an Firma: nein.

Erklärung zu nichtfinanziellen Interessen

Die Autorinnen/Autoren geben an, dass kein Interessenkonflikt besteht 


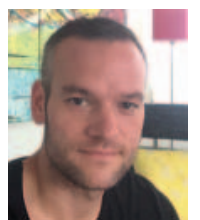

\section{Christoph Goltz}

Dr. med. Christoph Goltz, Facharzt für Orthopädie und Unfallchirurgie, arbeitet seit 2017 als hauptamtlicher Notarzt an den GRN-Kliniken am Standort Sinsheim. Zudem ist er Leitender Notarzt des Rettungsdienstbereichs RheinNeckar.

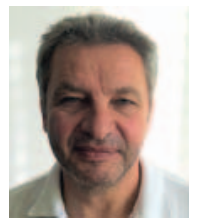

\section{Bernd Drechsler}

Bernd Drechsler arbeitet seit 32 Jahren als Rettungsassistent beim DRK Heidelberg.

\section{Korrespondenzadresse}

\section{Dr. med. Christoph Goltz}

GRN-Klinik Sinsheim

Alte Waibstadter Straße 2

74889 Sinsheim

Christoph.Goltz2@grn1.de
[1] Deutsche Reiterliche Vereinigung e. V. Bundesverband für Pferdesport und Pferdezucht Im Internet: Zahlen und Fakten. www.pferd-aktuell.de/fn-service/zahlen-fakten/zahlenfakten.html; Stand: 20.01.2019

[2] Sorli JM. Equestrian injuries: a five year review of hospital admissions in British Colombia, Canada. Inj Prev 2000; 6: 59-61

[3] Mayberry JC, Pearson TE, Wiger KJ et al. Equestrian injury prevention efforts need more attention to novice riders. J Trauma 2007; 62 (3): 735-739

[4] Schicho A, Einwag D, Gebhard F et al. Schwerverletzte durch Unfälle im Reitsport. Dtsch Z Sportmed 2014; 65: 258-261

[5] Fleischer LE, Faschingbauer M, Seide $\mathrm{K}$ et al. Verletzungsmuster bei Reitunfällen. Dtsch Z Sportmed 2016; 67: 270-275

[6] Havlik HS. Equestrian Sport-Related Injuries: A Review of Current Literature. Curr Sports Med Rep 2010; 9: 299-302

[7] Hebecker R, Piek J. Schädel-Hirn-Verletzungen durch Reitunfälle. Notfallmedizin 2003; 29: 304-309

[8] Schröter C, Wilhelmi M, Bielefeld D et al. Intensivmedizinische Behandlung von Patienten nach reitsportbedingten Unfällen. Notfall Rettungsmed 2018; 21: 608

Bibliografie

DOI https://doi.org/10.1055/a-0644-8143

retten 2020; 9: 32-39

(c) Georg Thieme Verlag KG, Stuttgart · New York

ISSN 2193-2387 


\section{Punkte sammeln auf CME.thieme.de}

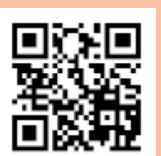

Diese Fortbildungseinheit ist in der Regel 12 Monate online für die Teilnahme verfügbar.

Den genauen Einsendeschluss finden Sie unter https://eref.thieme.de/CXB441D.

Sollten Sie Fragen zur Online-Teilnahme haben, finden Sie unter https://cme.thieme.de/hilfe

eine ausführliche Anleitung. Wir wünschen viel Erfolg beim Beantworten

der Fragen!

Unter https://eref.thieme.de/CXB441D oder über den QR-Code kommen Sie direkt zur Startseite des Wissenstests.

\section{Frage 1}

Welche Aussage trifft zu?

A Die überwiegende Zahl der verletzten Reiter ist männlich.

B Meist sind leicht verletzte Reiter über 45 Jahre alt.

C Etwa jeder 5. Reiter verletzt sich im Lauf seiner Reitkarriere ernsthaft.

D Einer von 1000000 Reitern stirbt pro Jahr durch einen Reitunfall.

E Die meisten schwer verletzten Reiter sind zwischen 8 und 18 Jahren alt.

\section{Frage 2}

Pferde...

A wiegen im Schnitt $150 \mathrm{~kg}$.

B sind Fluchttiere.

C sind max. $30 \mathrm{~km} / \mathrm{h}$ schnell.

D treten mit einer Kraft bis $500 \mathrm{~kg} z u$.

$\mathrm{E}$ treten nur, wenn man sich von vorne nähert und sie erschreckt.

\section{Frage 3}

Der häufigste Unfallmechanismus bei Reitunfällen ist ...
A ein Tritt des Pferdes.
$B$ ein Biss des Pferdes.
C das Einklemmen der Finger im Zaumzeug.
D der Anprall gegen ein Hindernis.
E der Sturz vom Pferd.

\section{Frage 4}

\section{Welche Aussage ist richtig?}

A Bei einem Sturz vom Pferd kommt es immer zu Mehrfachverletzungen.

B Bei Reitunfällen kommt es überwiegend zu Einzelverletzungen.

C Bei Reitunfällen lässt sich der Unfallmechanismus leicht rekonstruieren.

D Der Reiter kann den Sturz immer genau erklären.

E Der Standort des Pferdes nach dem Sturz gibt einen genauen Überblick über den Unfallmechanismus.

\section{Frage 5}

Bei einem Sturz vom Pferd ist am häufigsten folgende Körperregion verletzt:
A die oberen Extremitäten
B die Wirbelsäule
C der Fuß
D der Kopf
E das Abdomen

\section{Frage 6}

Welche Aussage ist richtig?

A Da Pferde scheue Fluchttiere sind, ist es wichtig, diese zunächst mithilfe des Martinshorns vom Unfallort zu vertreiben.

B Als erste Maßnahme wird der Patient laufend zum RTW geführt.

C Da dem Patienten nach dem Reiten sehr warm ist, wird er präklinisch gekühlt.

D Die Befragung von Zeugen kann wichtige Rückschlüsse auf den Unfallmechanismus geben.

E Die Immobilisation der Halswirbelsäule erfolgt mit einer weichen Halskrause.

\section{Frage 7}

In welchem Fall soll nach der S3-Leitlinie Polytrauma/Schwerverletzten-Behandlung der Deutschen Gesellschaft für Unfallchirurgie das Schockraumteam alarmiert werden?

A Isolierte, dislozierte Fraktur des Oberarms ohne Beeinträchtigung der peripheren Durchblutung, Motorik und Sensibilität.

B Sturz aus 3,5 m Höhe ohne erkennbare Traumafolgen.

C Amputation des Ringfingers durch einen Pferdebiss.

D Isoliertes Schädel-Hirn-Trauma mit einer GSC von 12 nach Sturz von einem stehenden Pferd aus ca. 2 m Höhe.

E Pferdetritt auf den Fuß mit beginnendem Kompartmentsyndrom.

- Weitere Fragen auf der folgenden Seite... 


\section{Punkte sammeln auf CME.thieme.de}

\section{Frage 8}

Ein Team Time-out während der Akutversorgung dient ...

A der kurzen Darstellung der Fakten und Befunde, Definition der Probleme und Festlegung der weiteren Maßnahmen.

B der Kommunikation mit den Angehörigen und der Polizei.

$C$ dem Zeitgewinn zum Anlegen von Verbänden und Schienen.

D der Besprechung des Unterschieds zwischen Pferd und Pony.

E der Überprüfung des Fachwissens der beteiligten Einsatzkräfte.

\section{Frage 9}

Welche Aussage ist richtig?

A Ein Pferdetritt gegen den Thorax führt nicht zur Verletzung innerer Organe.

B Bei einem Pferdetritt wirkt eine kleine Kraft auf eine große Fläche.

C Man nähert sich einem Pferd am sichersten langsam von vorne oder seitlich.

D Ein Pferdetritt in den Bauch führt nicht zur Verletzung innerer Organe.

E Pferde beißen niemals.

\section{Frage 10}

Was gehört nicht obligat zur Versorgung eines polytraumatisierten Reiters?
A Pulsoxymetrie
B Blutdruckmessung
C Venöser Zugang
D 12-Kanal-EKG
E Immobilisation 